\title{
Prognostic role of plasma mammaglobin A expression in breast carcinoma patients: a meta-analysis
}

This article was published in the following Dove Press journal:

OncoTargets and Therapy

\section{Yuanyuan $\mathrm{Hu}$ \\ Peipei Liu \\ DiWu \\ Youhong Jiang}

Molecular Oncology Laboratory of Cancer Research Institute, The First Affiliated Hospital of China Medical University, Shenyang, People's Republic of China
Correspondence: Youhong Jiang Molecular Oncology Laboratory of Cancer Research Institute, The First Affiliated Hospital of China Medical University, Shenyang I I000 I, People's Republic of China

Tel +86 I38 40380604

$\mathrm{Fax}+862483282473$

Email jiangyouhong2000@aliyun.com

\begin{abstract}
Mammaglobin A expression in peripheral blood (PB) of breast carcinoma patients has been evaluated by various studies, but the findings have been inconsistent. This metaanalysis aimed to clarify the prognostic value of mammaglobin A in the PB of breast carcinoma patients and define its relationships with clinicopathological features. PubMed, EMBASE, and the Cochrane Library databases were systematically searched for eligible studies through September 26, 2017. A total of 20 studies involving 2,323 patients were analyzed, and the data were independently extracted by two researchers. The combined hazard ratios (HRs) with 95\% CI was used to assess the association between survival data and plasma mammaglobin A expression, and odds ratios (ORs) and 95\% CIs were used to assess the associations between clinicopathological parameters and plasma mammaglobin A expression. The results indicated that plasma mammaglobin A expression was a predictor of poor prognosis for breast carcinoma patients, with an HR of $2.08(95 \% \mathrm{CI}=1.48-2.91 ; P<0.0001)$ for overall survival. Moreover, plasma mammaglobin A was significantly associated with lymph node metastasis $(\mathrm{OR}=2.00$; 95\% CI=1.17-3.45; $P=0.01)$ and advanced tumor stage $(\mathrm{OR}=3.01 ; 95 \% \mathrm{CI}=1.57-5.77$; $P=0.0009)$ in breast carcinoma patients. However, the results revealed that plasma mammaglobin A was not significantly associated with tumor size $(\mathrm{OR}=1.29 ; 95 \% \mathrm{CI}=0.46-3.66 ; P=0.63)$, tumor differentiation $(\mathrm{OR}=0.99 ; 95 \% \mathrm{CI}=0.63-1.57 ; P=0.97)$, menopausal status $(\mathrm{OR}=0.75$; $95 \% \mathrm{CI}=0.48-1.18 ; P=0.22)$, estrogen receptor status $(\mathrm{OR}=0.78 ; 95 \% \mathrm{CI}=0.44-1.36 ; P=0.38)$, progesterone receptor status $(\mathrm{OR}=0.76 ; 95 \% \mathrm{CI}=0.57-1.02 ; P=0.07)$, or human epidermal growth factor receptor 2 status $(\mathrm{OR}=1.12 ; 95 \% \mathrm{CI}=0.78-1.59 ; P=0.54)$. In conclusion, the results demonstrate that positive plasma mammaglobin A expression might serve as a biomarker of poor prognosis for breast carcinoma patients.
\end{abstract}

Keywords: plasma mammaglobin A, breast carcinoma, prognosis, meta-analysis

\section{Introduction}

Breast carcinoma is the most frequently diagnosed cancer in women. ${ }^{1}$ Approximately $30 \%$ of patients die from breast carcinoma, despite significant improvements in detection and treatment. One of the main causes of death is the formation of metastasis. ${ }^{2}$ Some studies indicate that, although tumor cells are absent in lymphatic nodes or bone marrow, circulating tumor cells (CTCs) still exist in the peripheral blood (PB) of patients. ${ }^{2-4}$ Breast carcinoma is a systemic disease in which tumor cells can spread to the blood and lymph system in early stages. ${ }^{5}$ Because it is relatively easy to obtain blood samples, PB samples were accepted as targets for detection and prognosis evaluation of breast carcinoma: several serum makers - CA153, CA27.29, HER2/neu - are common biomarkers in breast carcinoma. ${ }^{6,7}$ However, due to tumor heterogeneity, 
the expression of tumor biomarkers varies among breast carcinoma patients. ${ }^{8}$ A new biomarker with high sensitivity and specificity is critical for correctly diagnosing and assessing the prognosis of breast carcinoma.

Mammaglobin A, which is composed of 93 amino acids, was identified by Watson and Fleming ${ }^{9}$ using a differential polymerase chain reaction in 1996. It is homologous with the secretory protein family. Mammaglobin A is overexpressed in $40 \%-80 \%$ of primary and metastatic breast carcinoma cases. ${ }^{10-12}$ Due to its unique and universal expression properties, it was viewed as an attractive diagnostic marker for breast carcinoma. ${ }^{12,13}$ In 1999, it was reported that mammaglobin A mRNA expression was detected in the blood samples of $49 \%$ of metastatic breast carcinoma patients. ${ }^{14}$ Since then, several studies have indicated that plasma mammaglobin A could be used as a sensitive marker for breast carcinoma CTCs. ${ }^{15-17}$ Additionally, some studies have demonstrated the association between plasma mammaglobin A expression and clinicopathological prognostic parameters in breast carcinoma before surgery and/or chemotherapy. ${ }^{18-20}$ However, the prognostic role of plasma mammaglobin A in breast carcinoma is still being debated.

To the best of our knowledge, no meta-analyses have been conducted to evaluate the prognostic role of mammaglobin A. Therefore, in this study, we aimed to perform a meta-analysis to evaluate the relationships between plasma mammaglobin A expression and prognostic parameters in breast carcinoma patients.

\section{Materials and methods Data search strategy}

Our meta-analysis was completed in accordance with the Preferred Reporting Items for Systematic Reviews and Meta-Analyses (PRISMA) statement. PubMed, EMBASE, and Cochrane Library electronic databases were searched for eligible studies to incorporate into our meta-analysis. The search strategy was performed with the following terms: "mammaglobin" OR "mammaglobin A" OR "mammaglobin 1" OR "hMAM" OR "Mam-A" OR "MGB1" OR "secretoglobin Family 2A Member 2" and "breast cancer" OR "breast carcinoma" OR “breast tumor”'OR “breast tumour” OR "breastneoplasm” OR "mammary gland cancer" and "survival” OR "outcome” OR "prognosis" OR "prognostic" OR "mortality".

\section{Inclusion and exclusion criteria}

Eligible articles met the following criteria: 1) studies focused on mammaglobin A expression in PB of breast carcinoma patients; 2) studies provided relationships between plasma mammaglobin A and clinical prognostic indicators or patho- logical parameters; and 3) studies provided adequate data to directly calculate hazard ratios (HRs), odds ratios (ORs), and relative risks with $95 \%$ CIs or to indirectly calculate statistics with relevant software. Exclusion criteria were as follows: 1) duplicate articles, review articles, case reports, conference abstracts, and letters; 2) cell or animal experiments, non-English language articles, or unavailable full text; 3 ) inadequate data to calculate HRs with 95\% CIs; and 4) the Kaplan-Meier curve could not be extracted.

\section{Data extraction}

On the basis of the inclusion and exclusion criteria, two researchers ( $\mathrm{Hu}$ and $\mathrm{Liu}$ ) independently extracted data from eligible studies. Relevant information included the name of the first author, year of publication, number of patients, ages of patients, country, specimen, method of detection, follow-up time, and end points of each study. If articles only provided a Kaplan-Meier curve, Engauge Digitizer software, version 3.0, (http://digitizer.sourceforge.net) was used to extract the data and calculate the corresponding HR with $95 \%$ CI with the program file designed by Tierney et al. ${ }^{21}$

\section{Quality assessment}

Two researchers (Hu and Liu) independently evaluated the quality of all included studies using the Newcastle-Ottawa Scale (NOS) with a 9-point scoring system. ${ }^{22}$ If the NOS score was 6 or higher, the study was regarded to be of high quality. Any disagreements were resolved by discussion between the two researchers or by consulting a third researcher $(\mathrm{Wu})$.

\section{Statistical analysis}

Stata SE12.0 (Stata Corp, TX, USA) and Review Manager version 5.3 (Cochrane Collaboration, Oxford, UK) were used to analyze the data from the included studies. The correlations between plasma mammaglobin A expression level and indicators of cancer prognosis (such as overall survival [OS]) were evaluated with HRs and the corresponding $95 \%$ CIs. A pooled HR $>1$ indicated poor survival for breast carcinoma patients; a pooled $\mathrm{HR}<1$ indicated improved survival for breast carcinoma patients. Pooled ORs and their 95\% CIs were used to evaluate the correlations between plasma mammaglobin A expression and clinicopathological features of breast carcinoma, including tumor size; tumor stage; tumor differentiation; menopausal status; lymph node metastasis; and estrogen receptor (ER), progesterone receptor $(\mathrm{PR})$, and human epidermal growth factor receptor 2 (Her-2) statuses. The heterogeneity among the incorporated studies was detected by the $\mathrm{I}^{2}$ statistic or the chi-squared 
test. An $I^{2}>50 \%$ or a $P$-value $<0.10$ indicated significant heterogeneity among the studies. The pooled HR or OR was calculated with a fixed effects model if heterogeneity did not significantly exist in these studies; a random effects model was used if significant heterogeneity was observed. In order to explore the source of the heterogeneity, subgroup analyses or sensitivity analyses were performed. Funnel plots and Egger's tests were used to evaluate publication bias. $P$-values $<0.05$ were considered to indicate significant differences.

\section{Results}

\section{Search results and study characteristics}

Initially, 260 articles were identified using our search strategy. According to inclusion and exclusion criteria, a total of 20 articles $^{15,17-20,23-37}$ involving 2,323 breast carcinoma patients were included in the meta-analysis (Figure 1). Detailed characteristics of the included studies are summarized in Table 1: the publication years ranged from 2000 to 2016, and the number of patients in each study ranged from 48 to 321 . Of the 20 studies, seven reported OS of breast carcinoma patients, 16 reported lymph node metastasis, 10 reported tumor stage, 11 involved tumor differentiation, five involved tumor size, five mentioned menopausal status, 12 noted ER status, 11 noted PR status, and eight noted Her-2 status.

\section{Meta-analysis results}

Plasma mammaglobin A expression and OS

A total of seven studies reported correlations between plasma mammaglobin A expression and OS. The pooled HR and $95 \%$ CI were calculated by fixed effects model because heterogeneity was absent among these studies $\left(I^{2}=0 \% ; P=0.54\right.$; Figure 2$)$.

\section{PRISMA 2009 flow diagram}

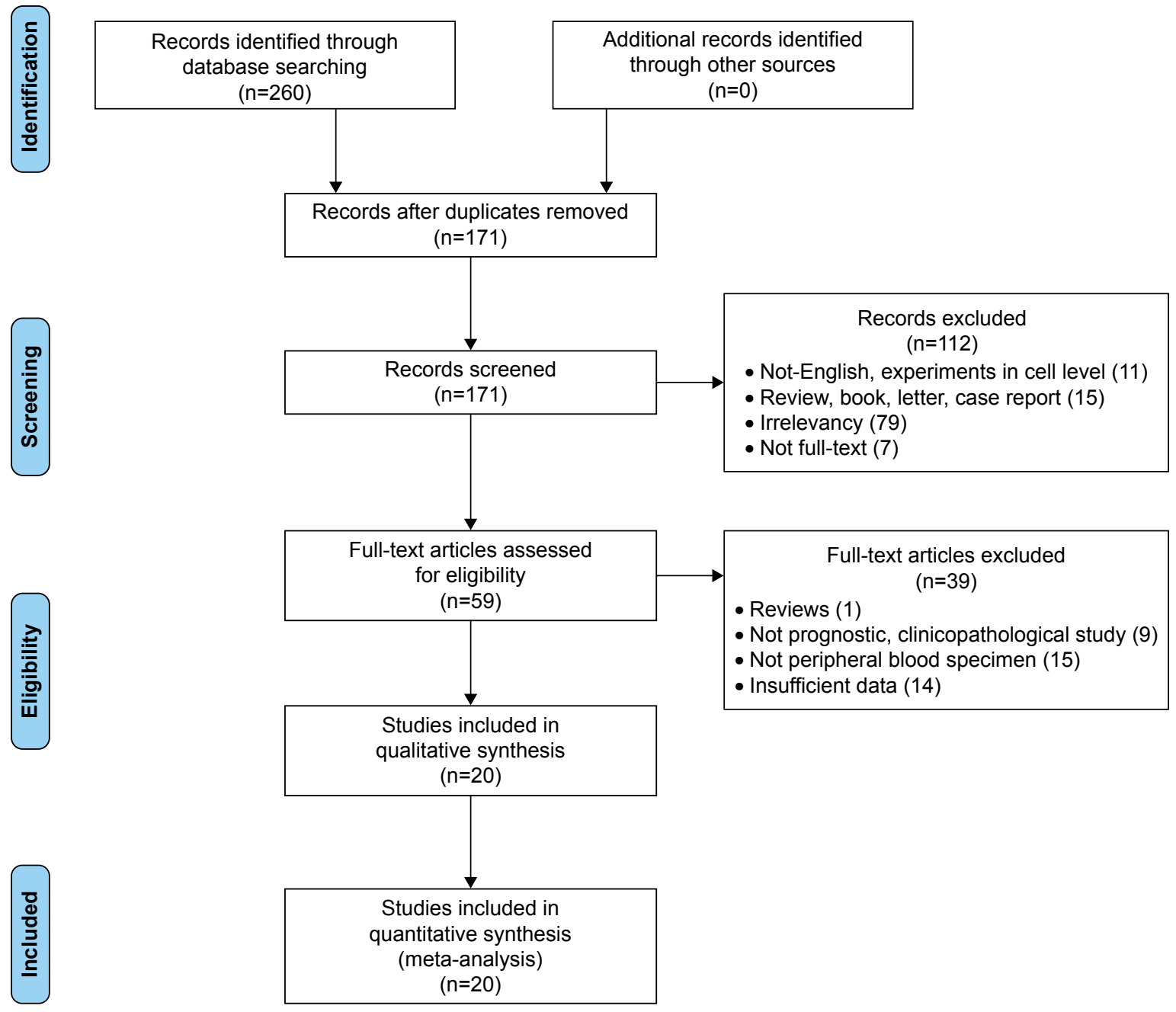

Figure I Flow diagram of study selection.

Note: Moher D, Liberati A, Tetzlaff J, Altman DG, The PRISMA Group (2009). Preferred Reporting Items for Systematic Reviews and Meta-Analyses: The PRISMA Statement. PLoS Med 6(7): el 000097 . doi: I0.137I/joumal.pmed I000097.48 
Table I Characteristics of the studies included in the meta-analysis

\begin{tabular}{|c|c|c|c|c|c|c|c|c|c|}
\hline Author & Year & Country & $\begin{array}{l}\text { Sample } \\
\text { size }\end{array}$ & Age, years & Specimen & $\begin{array}{l}\text { Detection } \\
\text { method }\end{array}$ & Follow-up (months) & End-point & $\begin{array}{l}\text { NOS } \\
\text { score }\end{array}$ \\
\hline Marques et $\mathrm{al}^{37}$ & 2009 & Portugal & 321 & Median: 5 I (22-8I) & PB & RT-PCR & median: 43 (3-74) & DFS & 7 \\
\hline \multirow[t]{2}{*}{$\mathrm{Li}$ and Zhang ${ }^{34}$} & 2016 & People's & 80 & NA & PB & RT-PCR & NA & NA & 5 \\
\hline & & $\begin{array}{l}\text { Republic of } \\
\text { China }\end{array}$ & & & & & & & \\
\hline El-Sharkawy et al ${ }^{36}$ & 2007 & Egypt & 48 & Range: $25-60$ & PB & Nested-RT-PCR & NA & NA & 5 \\
\hline Lee et $\mathrm{a}^{25}$ & 2012 & South Korea & 82 & Median: 50 (26-82) & PB & RT-PCR & median: 20.9 (I4.5-62.I) & OS/EFS & 8 \\
\hline Ntoulia et al ${ }^{17}$ & 2006 & Greece & 101 & Median: 53 (26-75) & PB & Nested-RT-PCR & median: 24 (I-80) & OS/DFI & 8 \\
\hline \multirow[t]{2}{*}{ Liu et $\mathrm{a}^{30}$} & 2012 & People's & 109 & Median: 54 (27-76) & PB & RT-PCR & NA & NA & 5 \\
\hline & & $\begin{array}{l}\text { Republic of } \\
\text { China }\end{array}$ & & & & & & & \\
\hline Lin et $\mathrm{al}^{29}$ & 2003 & Taiwan & 79 & Median: 47 (32-85) & PB & RT-PCR & NA & NA & 5 \\
\hline Ignatiadis et al ${ }^{15}$ & 2008 & Greece & 175 & Median: 54 (28-75) & PB & RT-PCR & NA & OS/DFS & 8 \\
\hline Chen et $\mathrm{a}^{28}$ & 2006 & Taiwan & 102 & NA & PB & membrane array & NA & NA & 6 \\
\hline Ceballos et al ${ }^{19}$ & 2011 & Argentina & 65 & Range: 29-80 & PB & Nested-RT-PCR & NA & NA & 6 \\
\hline \multirow[t]{2}{*}{ Zhao et $\mathrm{al}^{20}$} & 2013 & People's & 98 & Median: 53.2 (33-78) & PB & RT-PCR & NA & OS/PFS & 8 \\
\hline & & $\begin{array}{l}\text { Republic of } \\
\text { China }\end{array}$ & & & & & & & \\
\hline Reinholz et $\mathrm{al}^{24}$ & 2011 & America & 86 & NA & PB & $R T-q P C R$ & NA & OS/PFS & 7 \\
\hline Ferrucci et $\mathrm{a}^{23}$ & 2004 & Italy & 90 & Median: 46 (24-7I) & PB & RT-PCR & median: 36 & OS/RFS & 8 \\
\hline Bolke et $\mathrm{al}^{27}$ & 2009 & Germany & 63 & NA & PB & RT-PCR & NA & NA & 5 \\
\hline Ferro et $\mathrm{al}^{18}$ & 2010 & Italy & 190 & Median: 63.2 (33-93) & PB & RT-PCR & NA & NA & 6 \\
\hline Cerveira et $\mathrm{al}^{35}$ & 2004 & Portugal & 54 & NA & PB & RT-PCR & NA & NA & 5 \\
\hline Grunewald et $\mathrm{a}^{31}$ & 2000 & Austria & 133 & NA & PB & Nested-RT-PCR & NA & NA & 6 \\
\hline Zehentner et $\mathrm{al}^{32}$ & 2004 & USA & 84 & NA & PB & Real-time-RT-PCR & NA & NA & 6 \\
\hline Mikhitarian et $\mathrm{al}^{33}$ & 2008 & USA & 215 & Median: 56.3 (29-84) & PB & Real-time-RT-PCR & NA & NA & 6 \\
\hline Benoy et $\mathrm{a}^{26}$ & 2006 & Belgium & 148 & Range: $27-88$ & PB & Real-time-RT-PCR & median: 28.5 & OS & 7 \\
\hline
\end{tabular}

Note: Data in parenthesis represent ranges.

Abbreviations: NA, not available; PB, peripheral blood; DFS, disease-free survival; OS, overall survival; EFS, event-free survival; DFI, disease-free interval; PFS, progressionfree survival; RFS, relapse-free survival; NOS, Newcastle-Ottawa Scale; RT-qPCR, reverse transcription/real-time, quantitative PCR.

The expression of plasma mammaglobin A was significantly associated with poor OS of breast carcinoma patients (pooled $\mathrm{HR}=2.08$; 95\% CI=1.48-2.91; $P<0.0001$; Figure 2).

\section{Plasma mammaglobin A expression} and clinicopathological parameters

Pooled ORs were used to define the relationships between plasma mammaglobin A expression and clinicopathological parameters of breast carcinoma. The results of random effects models showed that plasma mammaglobin
A expression was significantly correlated with lymph node metastasis and tumor stage (positive vs negative: $\mathrm{OR}=2.00$; 95\% CI=1.17-3.45; $P=0.01 ; I^{2}=73 \%$; stage III-IV vs I-II: $\mathrm{OR}=3.01 ; 95 \% \mathrm{CI}=1.57-5.77 ; P=0.0009 ; \mathrm{I}^{2}=65 \%$; Figure 3 ). However, plasma mammaglobin A expression was not correlated with other clinicopathological parameters, including menopausal status (premenopausal vs postmenopausal: $\mathrm{OR}=0.75 ; 95 \% \mathrm{CI}=0.48-1.18 ; P=0.22 ; I^{2}=0 \%$ ), tumor differentiation (poorly defined vs well-to-moderately defined: $\mathrm{OR}=0.99 ; 95 \% \mathrm{CI}=0.63-1.57 ; P=0.97 ; I^{2}=44 \%$ ), tumor size

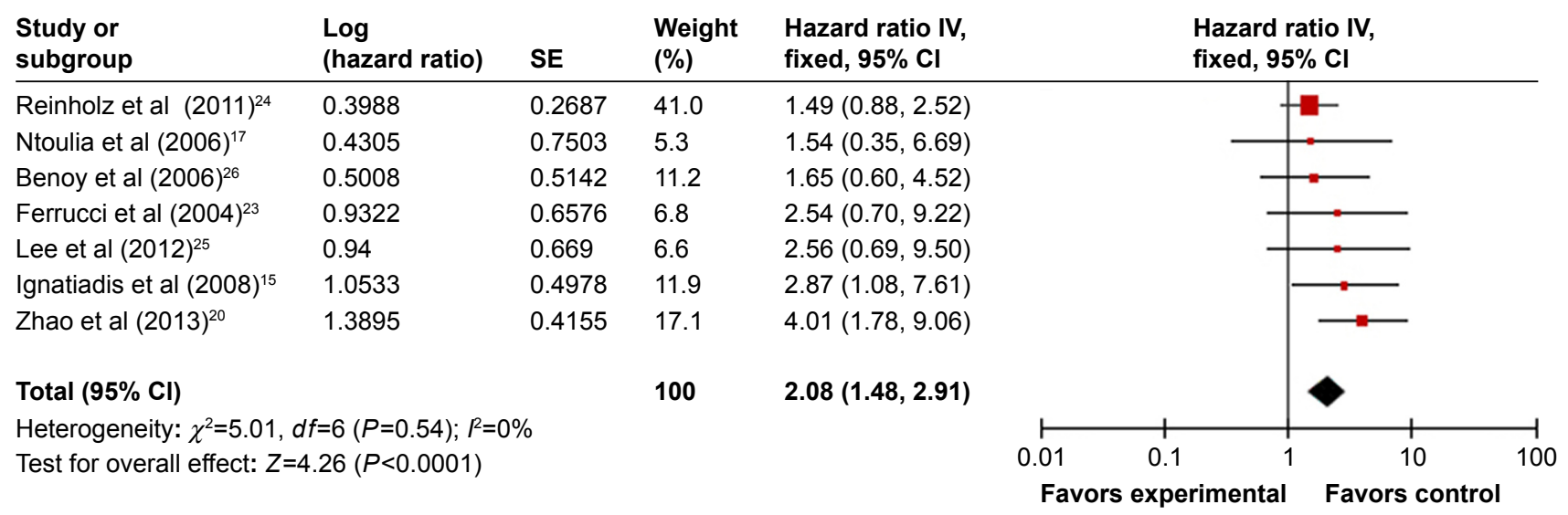

Figure 2 A Forest plot of the association between plasma mammaglobin. A expression and overall survival in breast carcinoma patients. Abbreviation: SE, standard error. 


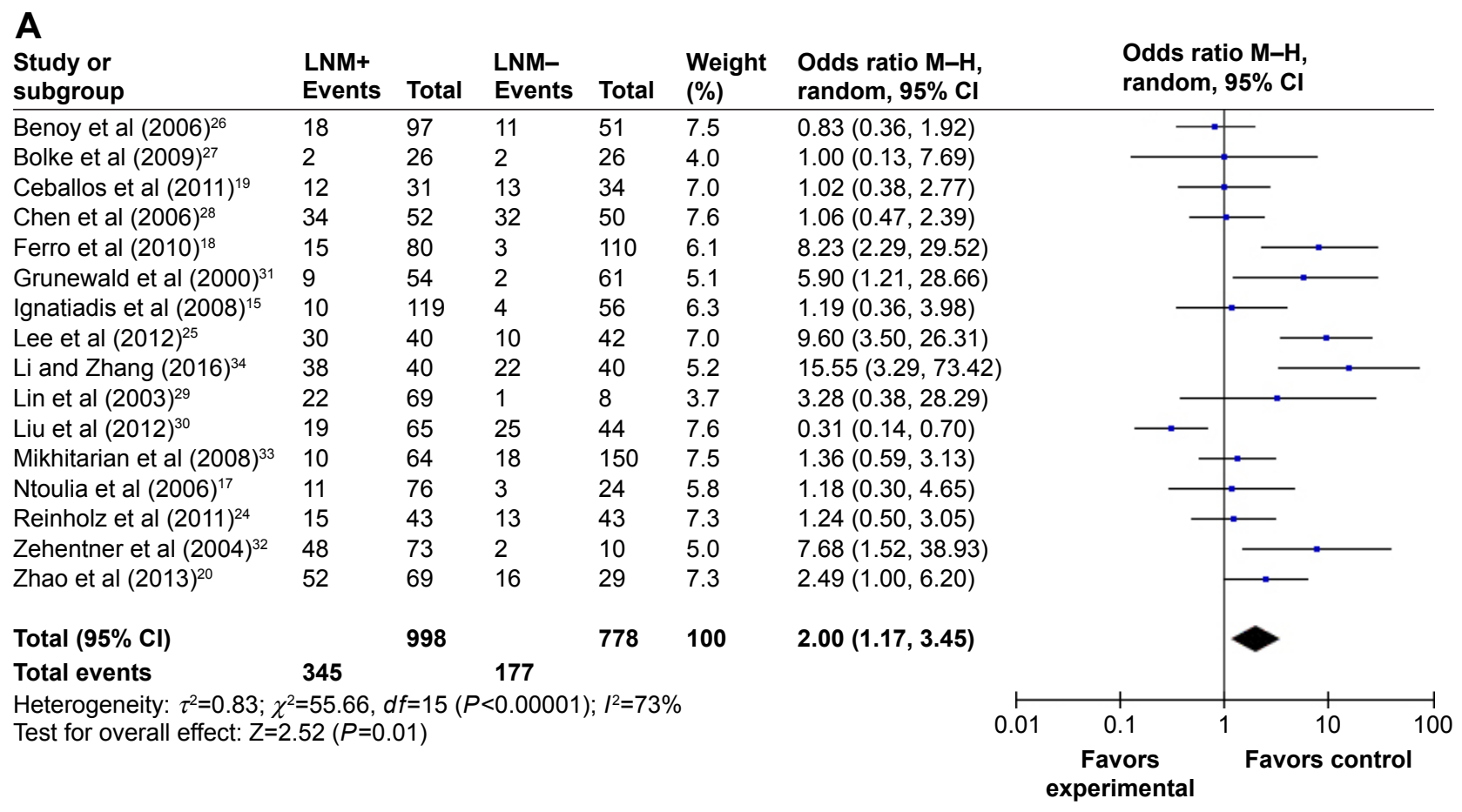

B

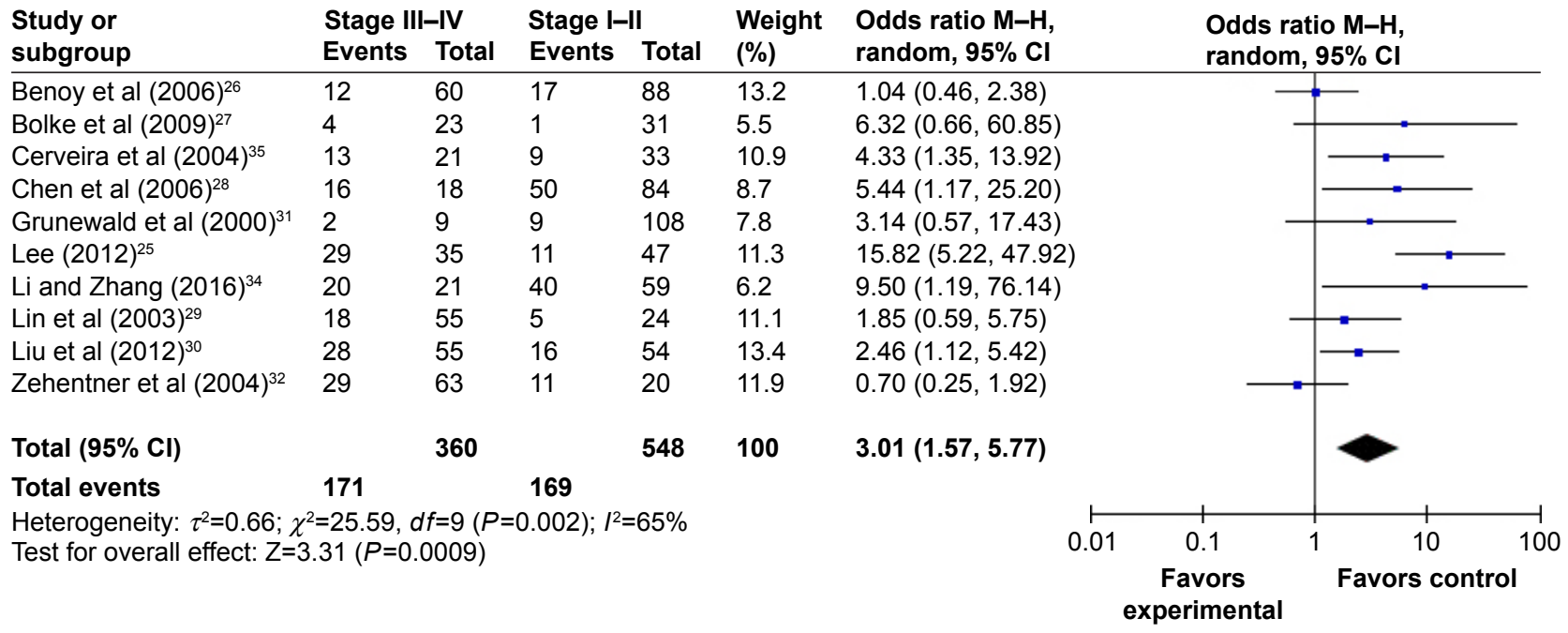

Figure 3 Forest plots of ORs for the associations between plasma mammaglobin A expression and (A) lymph node metastasis and (B) tumor stage. Abbreviations: ORs, odds ratios; LNM, lymph node metastasis.

$(>2 \mathrm{~cm}$ vs $\leq 2 \mathrm{~cm}: \mathrm{OR}=1.29 ; 95 \% \mathrm{CI}=0.46-3.66 ; P=0.63$; $I^{2}=78 \%$ ), ER status (ER-positive vs ER-negative: $\mathrm{OR}=0.78$; 95\% CI $=0.44-1.36 ; P=0.38 ; I^{2}=69 \%$ ), PR status (PR-positive vs $P R$-negative: $\mathrm{OR}=0.76 ; 95 \% \mathrm{CI}=0.57-1.02 ; P=0.07$; $I^{2}=0 \%$ ), and Her-2 status (Her-2-positive vs Her-2-negative: $\mathrm{OR}=1.12 ; 95 \% \mathrm{CI}=0.78-1.59 ; P=0.54 ; I^{2}=30 \%$ ) (Figure 4).

Furthermore, significant heterogeneity was detected in the meta-analysis of the correlations between plasma mammaglobin A expression and lymph node metastasis and tumor stage. Therefore, a subgroup analysis was performed on the basis of ethnicity (Asian vs non-Asian). In the subgroup analysis of the correlation between plasma mammaglobin A expression and lymph node metastasis, the pooled OR $(95 \%$ CI) was 2.51 (0.75-8.41) for Asian patients and 1.72 (1.032.86) for non-Asian patients; subgroup differences were not observed $(P=0.57)$. Additionally, in the subgroup analysis of the correlation between plasma mammaglobin A expression and tumor stage, the pooled OR (95\% CI) was 4.68 (1.9711.16) for Asian patients and $1.81(0.80-4.08)$ for non-Asian patients; subgroup differences were not observed $(P=0.12)$.

\section{Sensitivity analysis and publication bias}

To assess the reliability of the results, a sensitivity analysis was performed by omitting individual studies. As shown in 


\section{A}

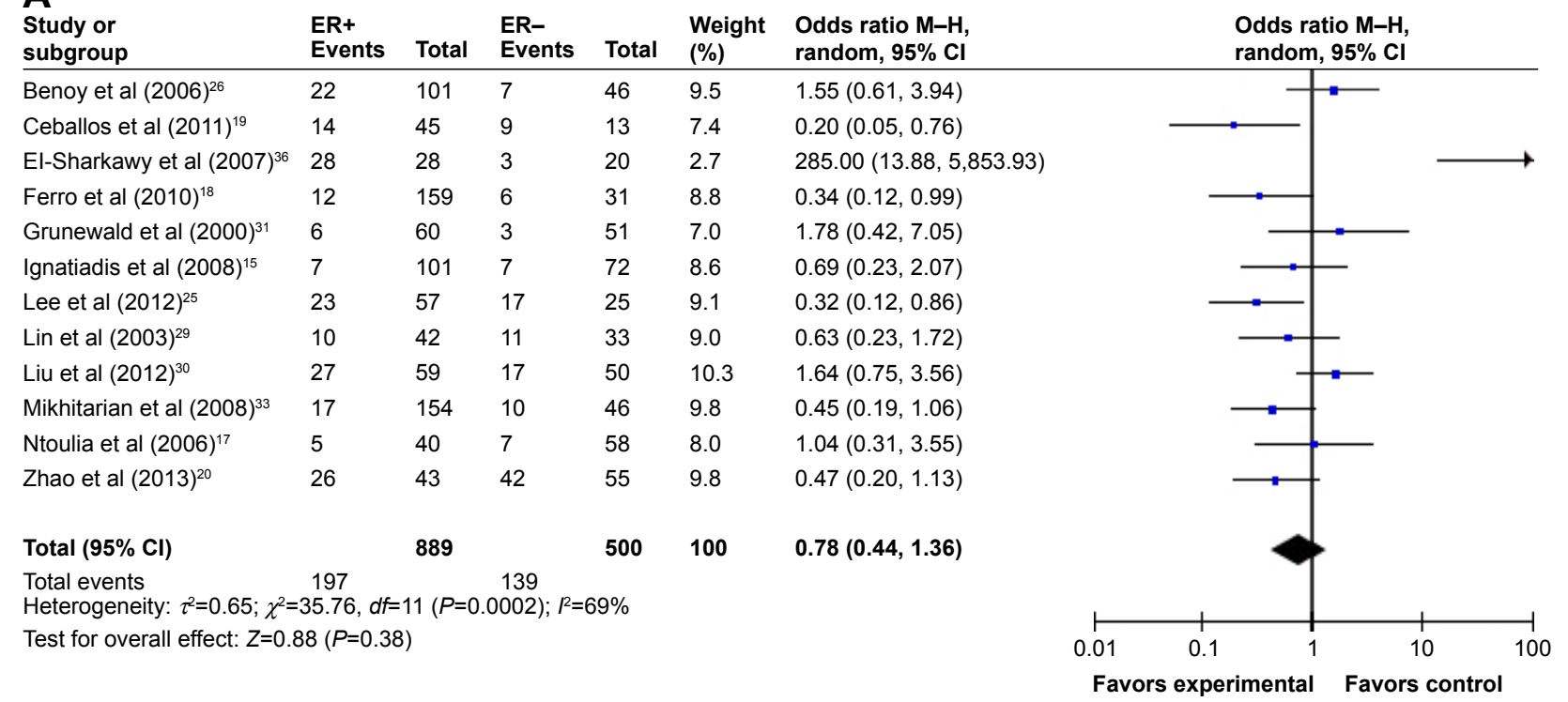

\section{B}

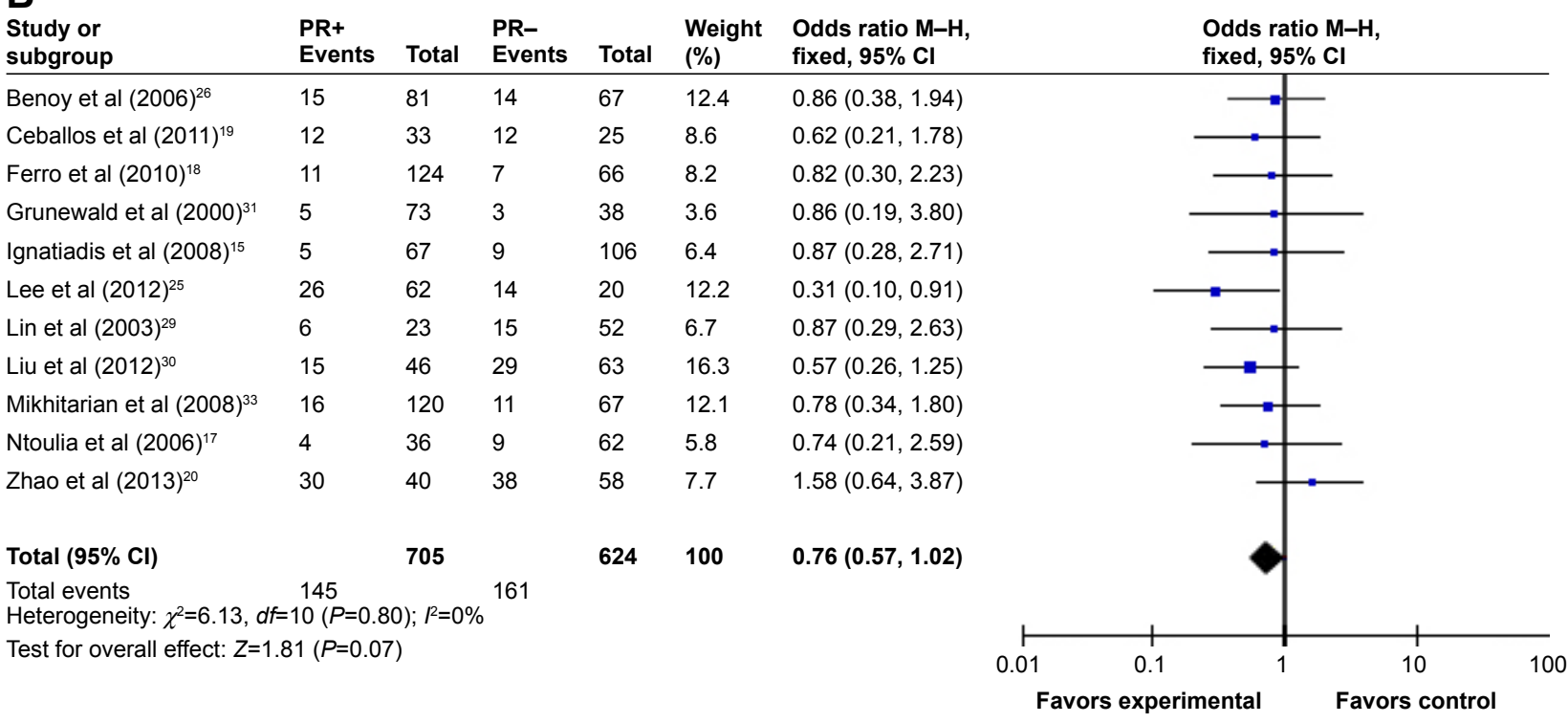

C

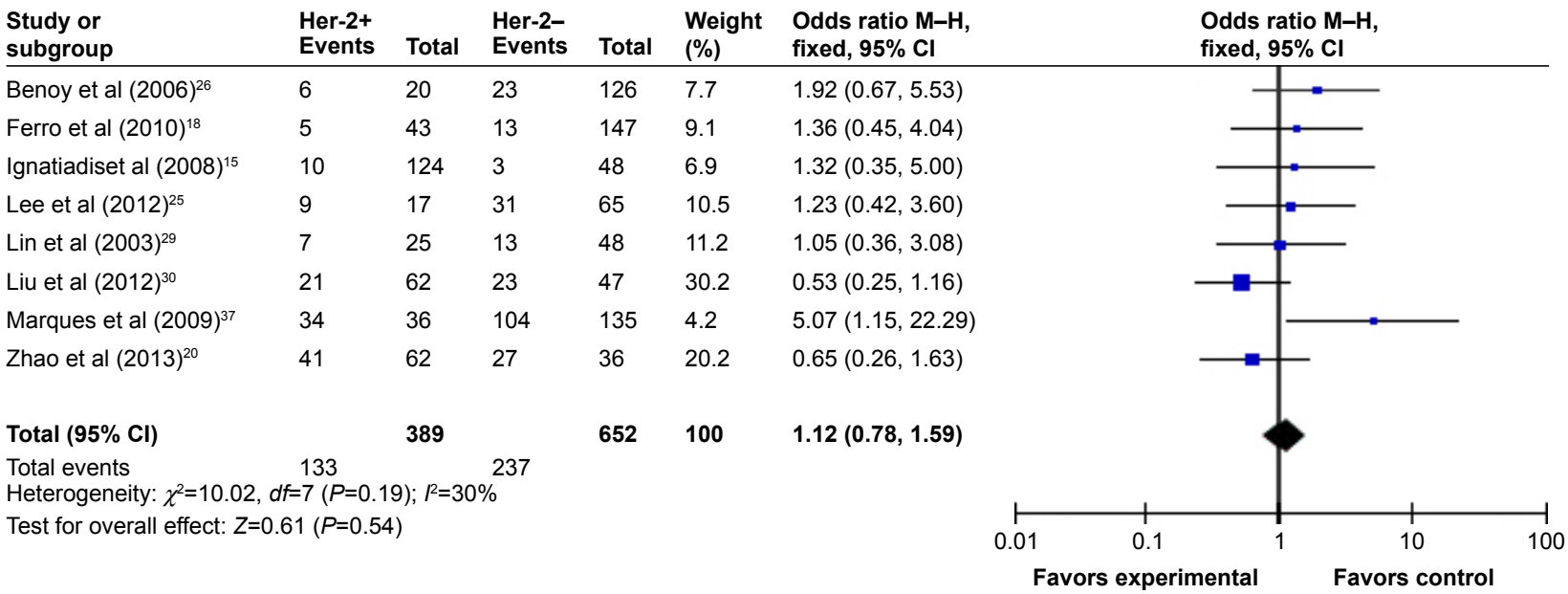

Figure 4 (Continued) 


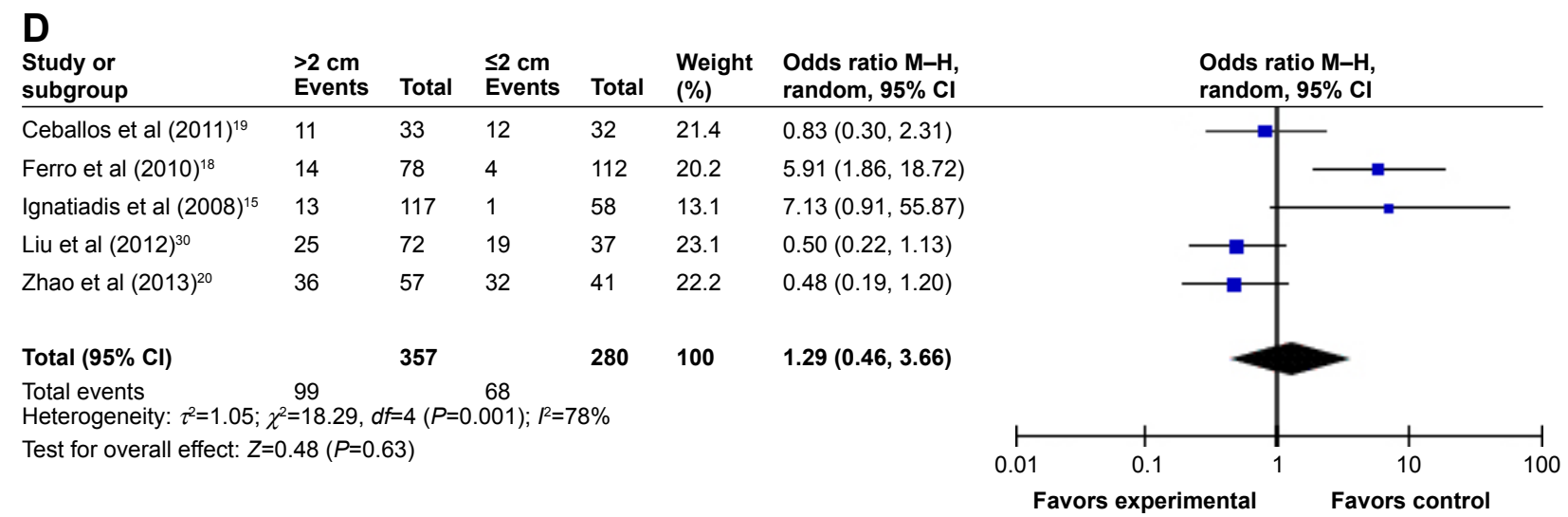

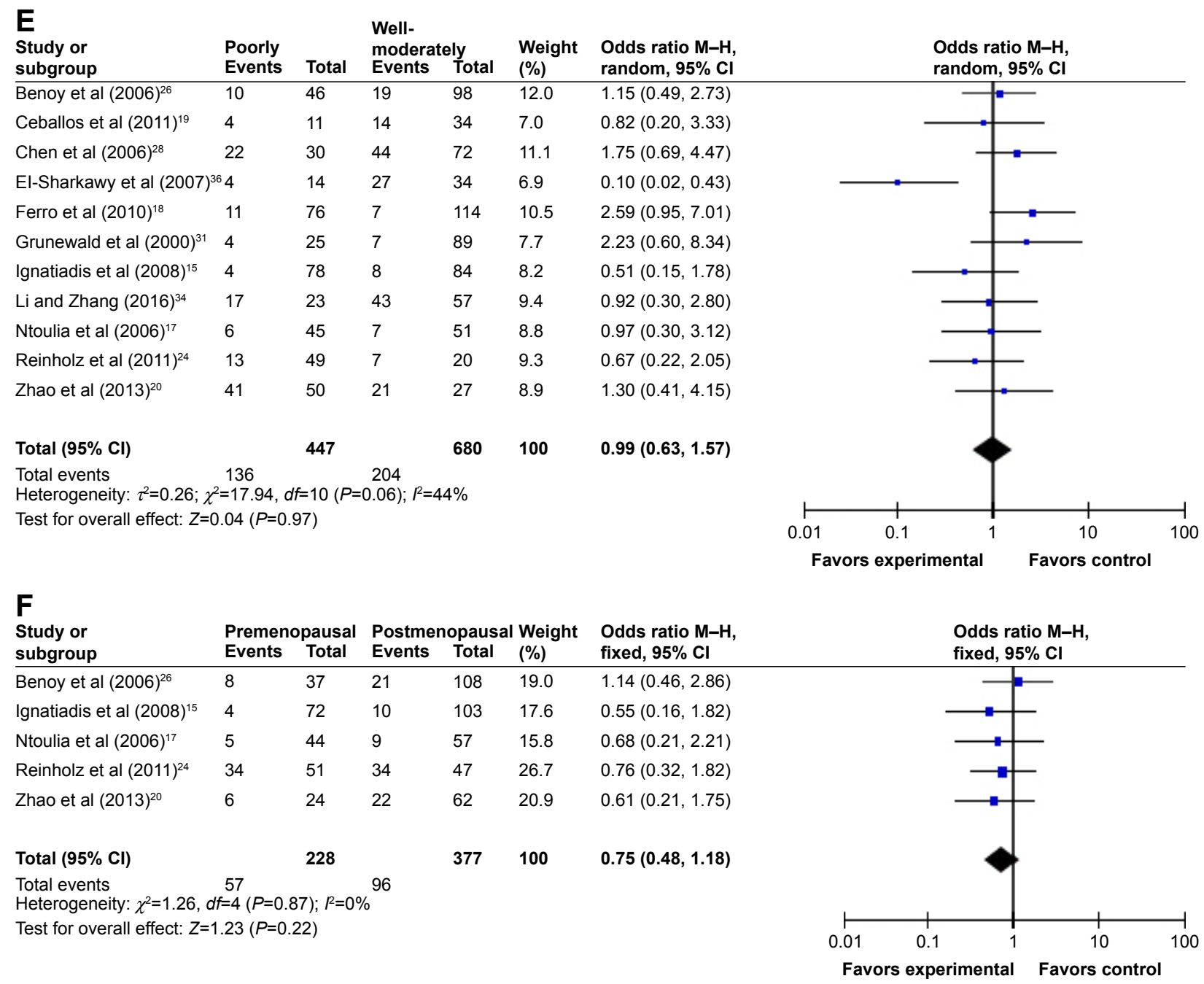

Figure 4 Forest plots of ORs for the associations between plasma mammaglobin A expression and other clinicopathological parameters: (A) plasma mammaglobin A expression and ER status; (B) plasma mammaglobin A expression and PR status; (C) plasma mammaglobin A expression and human epidermal growth factor receptor 2 (Her-2) status; (D) plasma mammaglobin A expression and tumor size; (E) plasma mammaglobin $A$ expression and tumor differentiation; and (F) plasma mammaglobin $A$ expression and menopausal status.

Abbreviations: ORs, odds ratios; ER, estrogen receptor; PR, progesterone receptor.

Figure 5, the pooled OR was not markedly affected by the removal of studies. Funnel plots and Egger's tests were used to evaluate potential publication bias in the included studies. When a meta-analysis included at least 10 articles, a funnel plot was used to evaluate publication bias. Therefore, the potential publication bias was evaluated related to plasma mammaglobin A expression and lymph node metastasis (Figure 6). According to the symmetry of the funnel plot and the result of Egger's test, publication bias was present in this meta-analysis $(P=0.040)$. 
A

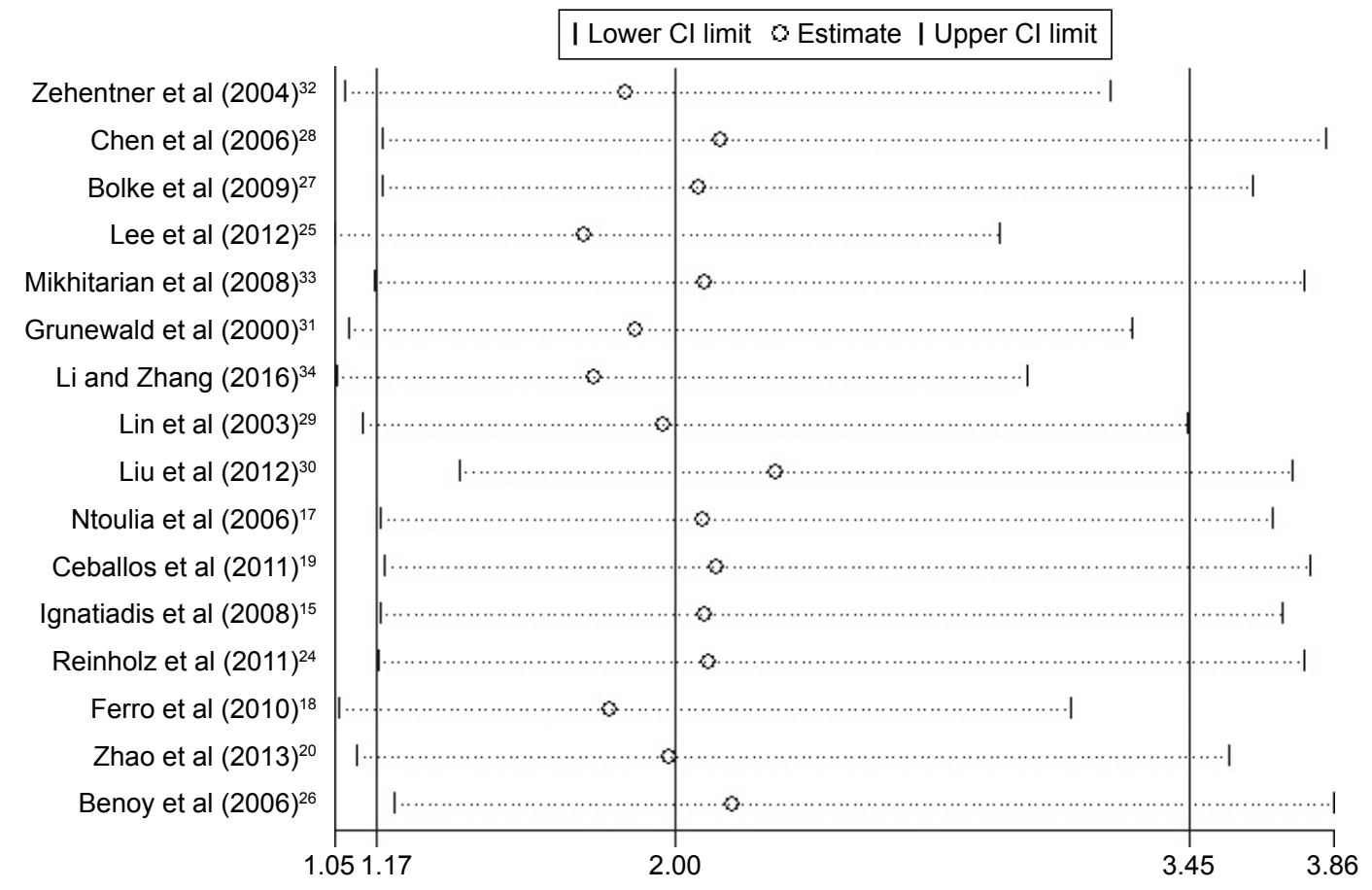

B

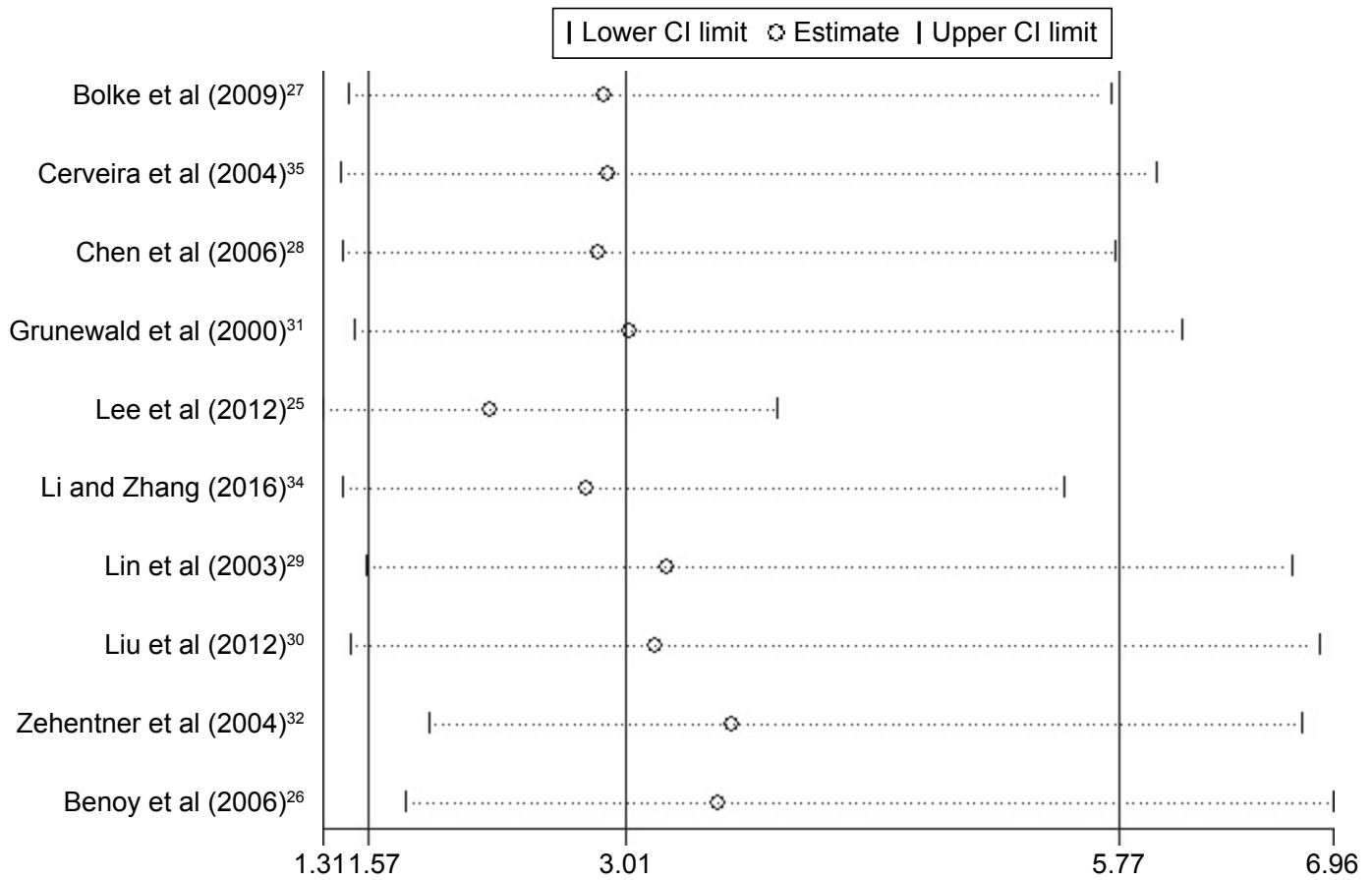

Figure $\mathbf{5}$ Sensitivity analysis for (A) lymph node metastasis and (B) tumor stage.

\section{Discussion}

Despite great advances in the early diagnosis and treatment of breast carcinoma, $\sim 50 \%$ of localized breast carcinoma and $30 \%$ of axillary lymph node-negative disease develop metastasis. ${ }^{38}$ Detection of serum tumor markers has been used as a non-invasive tool to diagnose and assess breast carcinoma. Mammaglobin A, composed of 93 amino acids, is specifically expressed in breast carcinoma. Some studies demonstrated that mammaglobin A expression was present in the blood samples of breast carcinoma patients, but it was absent in blood samples from patients with benign breast disease and healthy donors. ${ }^{18,20}$ The high specificity and 


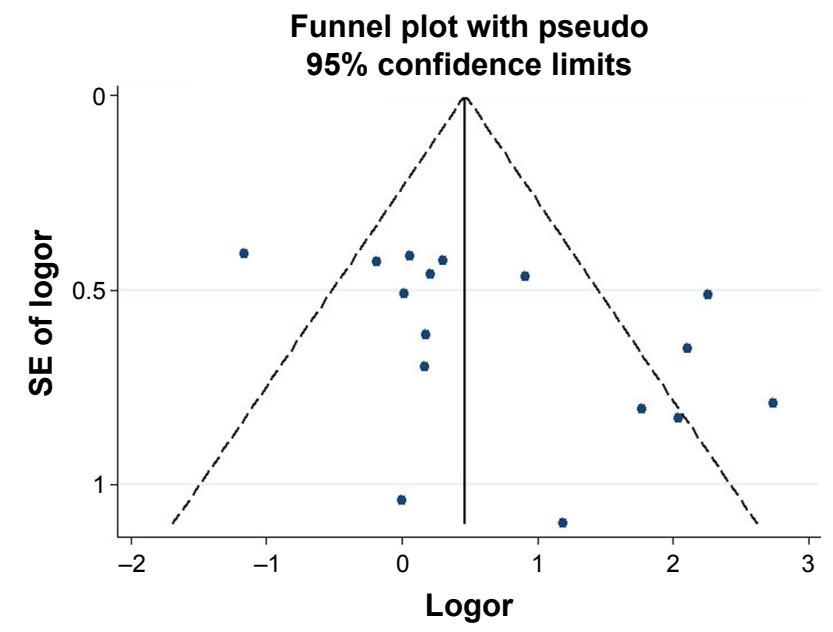

Figure 6 Funnel plot for analysis of plasma mammaglobin A expression and lymph node metastasis in breast carcinoma patients.

Abbreviation: SE, standard error.

sensitivity of plasma mammaglobin A made it a potential target for breast carcinoma cell detection in blood samples. As such, the mammaglobin A aptamer (molecular recognition probe) was used to detect circulating breast tumor cells and possessed better potential as a detection tool. ${ }^{39}$ Similarly, MamAb-680 (a targeted imaging probe) was structured through conjugating a near-infrared fluorescent dye and a mammaglobin A specific monoclonal antibody. In mice tumor models, MamAb-680 fluorescence imaging showed high sensitivity to detect breast carcinoma cells in axillary lymph node, which offered a noninvasive method to validate breast carcinoma staging. ${ }^{40}$ However, controversial and inconsistent conclusions related to the prognostic value of mammaglobin A in breast carcinoma have been reported. Lee et $a^{25}$ reported that plasma mammaglobin A was correlated with poor prognosis and shorter event-free survival in breast carcinoma patients: plasma mammaglobin A was significantly correlated with ER-negative/PR-negative status, advanced tumor stage, lymph node metastasis, and high histologic and nuclear grades. However, El-Sharkawy et $\mathrm{al}^{36}$ reported the contradictory finding that plasma mammaglobin A was correlated with a better prognosis: plasma mammaglobin A was significantly increased in breast carcinoma patients with low-grade tumors, ER-positive status, and a low Ki-67 proliferation index. As far as we know, no meta-analysis has yet been conducted to evaluate the prognostic value of plasma mammaglobin A in breast carcinoma patients, and our meta-analysis was the first to assess this topic.

In our meta-analysis, seven studies were incorporated to evaluate the correlation between plasma mammaglobin A expression and OS in breast carcinoma patients. Our results demonstrated that plasma mammaglobin A was significantly correlated with unfavorable OS in breast carcinoma patients. To further explore the effect of plasma mammaglobin A in breast carcinoma patients, we investigated the associations between plasma mammaglobin A and other clinicopathological parameters of breast carcinoma. We found that plasma mammaglobin A was significantly correlated with the presence of lymph node metastasis and with tumor stage. Plasma mammaglobin A expression in patients with lymph node metastasis was two-times higher than in patients without lymph node metastasis. Plasma mammaglobin A expression in patients with stage III-IV disease was 3.01-times higher than in patients with stage I-II disease. Thus, plasma mammaglobin A can be regarded as a valuable marker for evaluating advanced tumor stage and lymphatic metastasis. Poor prognosis is correlated with advanced clinical stage and lymphatic metastasis, and identifying lymphatic metastasis is critical for choosing postsurgical treatment. Regarding the biological role of mammaglobin A, Picot et al ${ }^{41}$ demonstrated that mammaglobin A mediated tumorigenesis and aggressiveness by increasing tumor cell proliferation, migration, and invasion. Concomitantly, mammaglobin A activated several signaling pathways, such as MAPK, FAK, MMPs, and NFkB, that were involved in cancer progression. Moreover, the inhibition of mammaglobin A expression resulted in the suppression of ZEB1, Snail, and Twist, which are mesenchymal-related genes. Consequently, mammaglobin A can regulate epithelial to mesenchymal (EMT) features, further confirming the biological significance of mammaglobin A in breast carcinome progression. Combined with our results, plasma mammaglobin A was a negative prognostic parameter and could serve as an indicator for clinical assessment in breast carcinoma patients.

In addition to being a potential prognostic indicator, mammaglobin $\mathrm{A}$ is a novel therapeutic target for breast carcinoma. The mammaglobin A promoter could be used as a biological tool to regulate target gene expression. IL-2, a cytokine, could exert an antitumor effect and was used in clinical treatment of some malignant tumors. However, a major treatment challenge was IL-2-associated toxicities. The mammaglobin A promoter/enhancer (MPE2) targeting expression of IL-2 to breast carcinoma cells was a feasible strategy for enhancing antitumor activity and minimizing toxicities. ${ }^{42}$ Similarly, the mammaglobin A promoter, coupled with an apoptosis gene, may offer a precise and advanced killing strategy for breast carcinoma ${ }^{43}$ However, beyond that, the membraneassociated mammaglobin A was used as a potential molecular target for drug delivery. ${ }^{44}$ Because of high specificity and immunogenicity, mammaglobin A was an attractive antigen 
for breast carcinoma vaccine therapy. In phase I clinical trials, mammaglobin A DNA vaccine was capable of effectively inducing $\mathrm{CD}^{+} \mathrm{T}$-cells and $\mathrm{CD} 4+\mathrm{ICOS}^{\mathrm{hi}} \mathrm{T}$-cells immune responses and improving progression free survival (PFS) of breast carcinoma patients, without significant adverse effects. ${ }^{45-47}$ These strategies provided a theoretical basis for subsequent mammaglobin A target therapy.

Statistical heterogeneity was present in the studies included in the meta-analysis. We used an ethnicity-based subgroup analysis to identify the source of heterogeneity. However, significant differences were not found in the subgroup analysis. In addition, a sensitivity analysis was conducted to detect the reliability of the results. We found that reliability of the results was not markedly influenced by omission of any individual study. Therefore, other factors, such as the number of patients, the ages of the patients, the method of detection, or follow-up time, may be the source of the heterogeneity. Additionally, the relationships between plasma mammaglobin A expression and other clinicopathological parameters (ie, menopausal status, tumor differentiation, tumor size, ER status, PR status, Her-2 status) were not statistically significant in breast carcinoma patients. To assess potential publication bias, we constructed a funnel plot and performed Egger's test for the relationship between plasma mammaglobin A expression and lymph node metastasis. The funnel plot revealed asymmetry, and Egger's test indicated that publication bias was present.

Some limitations of our meta-analysis should be taken into consideration. First, if the source of the heterogeneity was not found, it could affect the pooled results. This heterogeneity may derive from different patient characteristics and different study designs. Second, only articles written in English were included in the current meta-analysis, so language bias cannot be ruled out. Third, for studies without HRs with 95\% CIs, we recalculated data from KaplanMeier curves, which might weaken the reliability of the pooled results. Fourth, research that reports positive results is published more often than research that reports negative results, so the pooled results may be overestimations of the relationships among the biomarker and clinicopathological features. Finally, the small number of samples might weaken our conclusions. Thus, to obtain a more reliable conclusion, larger and more standardized studies are required in the future.

\section{Conclusion}

In conclusion, our meta-analysis demonstrated that plasma mammaglobin A expression was significantly correlated with poor OS, the presence of lymph node metastases, and advanced tumor stage in breast carcinoma patients. As such, plasma mammaglobin A is a potential marker of poor prognosis for breast carcinoma patients. In the future, larger studies should be conducted to confirm the prognostic value of plasma mammaglobin A expression in breast carcinoma patients.

\section{Disclosure}

The authors report no conflicts of interest in this work.

\section{References}

1. Siegel RL, Miller KD, Jemal A. Cancer statistics, 2016. CA Cancer J Clin. 2016;66:7-30.

2. Lacroix M. Significance, detection and markers of disseminated breast cancer cells. Endocr Relat Cancer. 2006;13:1033-1067.

3. Cristofanilli M, Hayes DF, Budd GT, et al. Circulating tumor cells: a novel prognostic factor for newly diagnosed metastatic breast cancer. J Clin Oncol. 2005;23:1420-1430.

4. Müller V, Stahmann N, Riethdorf S, et al. Circulating tumor cells in breast cancer: correlation to bone marrow micrometastases, heterogeneous response to systemic therapy and low proliferative activity. Clin Cancer Res. 2005;11:3678-3685.

5. Stathopoulou A, Vlachonikolis I, Mavroudis D, et al. Molecular detection of cytokeratin-19-positive cells in the peripheral blood of patients with operable breast cancer: evaluation of their prognostic significance. J Clin Oncol. 2002;20:3404-3412.

6. Van Poznak C, Somerfield MR, Bast RC, et al. Use of biomarkers to guide decisions on systemic therapy for women with metastatic breast cancer: American Society of Clinical Oncology clinical practice guideline. J Clin Oncol. 2015;33:2695-2704.

7. Carney WP, Bernhardt D, Jasani B. Circulating HER2 extracellular domain: a specific and quantitative biomarker of prognostic value in all breast cancer patients? Biomark Cancer. 2013;5:31-39.

8. Sauter ER. Reliable biomarkers to identify new and recurrent cancer. Eur J Breast Health. 2017;13:162-167.

9. Watson MA, Fleming TP. Mammaglobin, a mammary-specific member of the uteroglobin gene family, is overexpressed in human breast cancer. Cancer Res. 1996;56:860-865.

10. Wang Z, Spaulding B, Sienko A, et al. Mammaglobin, a valuable diagnostic marker for metastatic breast carcinoma. Int J Clin Exp Pathol. 2009;2:384-389.

11. Sasaki E, Tsunoda N, Hatanaka Y, Mori N, Iwata H, Yatabe Y. Breastspecific expression of MGB1/mammaglobin: an examination of 480 tumors from various organs and clinicopathological analysis of MGB1positive breast cancers. Mod Pathol. 2007;20:208-214.

12. Luo MH, Huang YH, Ni YB, et al. Expression of mammaglobin and gross cystic disease fluid protein-15 in breast carcinomas. Hum Pathol. 2013;44:1241-1250.

13. Fritzsche FR, Thomas A, Winzer KJ, et al. Co-expression and prognostic value of gross cystic disease fluid protein 15 and mammaglobin in primary breast cancer. Histol Histopathol. 2007;22:1221-1230.

14. Zach O, Kasparu H, Krieger O, Hehenwarter W, Girschikofsky M, Lutz D. Detection of circulating mammary carcinoma cells in the peripheral blood of breast cancer patients via a nested reverse transcriptase polymerase chain reaction assay for mammaglobin mRNA. J Clin Oncol. 1999;17:2015-2019.

15. Ignatiadis M, Kallergi G, Ntoulia M, et al. Prognostic value of the molecular detection of circulating tumor cells using a multimarker reverse transcription-PCR assay for cytokeratin 19, mammaglobin A, and HER2 in early breast cancer. Clin Cancer Res. 2008;14:2593-2600.

16. Roncella S, Ferro P, Bacigalupo B, et al. Human mammaglobin mRNA is a reliable molecular marker for detecting occult breast cancer cells in peripheral blood. J Exp Clin Cancer Res. 2005;24:265-271. 
17. Ntoulia M, Stathopoulou A, Ignatiadis M, et al. Detection of mammaglobin A-mRNA-positive circulating tumor cells in peripheral blood of patients with operable breast cancer with nested RT-PCR. Clin Biochem. 2006;39:879-887.

18. Ferro P, Franceschini MC, Bacigalupo B, et al. Detection of circulating tumour cells in breast cancer patients using human mammaglobin RTPCR: association with clinical prognostic factors. Anticancer Res. 2010; 30:2377-2382.

19. Ceballos MP, Zumoffen C, Massa E, et al. Detection of mammaglogin A in blood from breast cancer patients, before and after treatment, using a one-tube nested PCR protocol. Association with the absence of tumor estrogen receptors. Clin Biochem. 2011;44:1429-1433.

20. Zhao S, Yang H, Zhang M, et al. Circulating tumor cells (CTCs) detected by triple-marker EpCAM, CK19, and hMAM RT-PCR and their relation to clinical outcome in metastatic breast cancer patients. Cell Biochem Biophys. 2013;65:263-273.

21. Tierney JF, Stewart LA, Ghersi D, Burdett S, Sydes MR. Practical methods for incorporating summary time-to-event data into meta-analysis Trials. 2007;8:16.

22. Stang A. Critical evaluation of the Newcastle-Ottawa scale for the assessment of the quality of nonrandomized studies in meta-analyses. Eur J Epidemiol. 2010;25:603-605.

23. Ferrucci PF, Rabascio C, Mazzetta C, et al. Mammaglobin expression in leukapheresis products is a predictive marker of poor prognosis in women with high-risk breast cancer. Clin Cancer Res. 2004;10: 6039-6046.

24. Reinholz MM, Kitzmann KA, Tenner K, et al. Cytokeratin-19 and mammaglobin gene expression in circulating tumor cells from metastatic breast cancer patients enrolled in North Central Cancer Treatment Group trials, N0234/336/436/437. Clin Cancer Res. 2011;17:7183-7193.

25. Lee GW, Kim JY, Koh EH, et al. Plasma human mammaglobin mRNA associated with poor outcome in patients with breast cancer. Genet Mol Res. 2012;11:4034-4042.

26. Benoy IH, Elst H, Philips M, et al. Real-time RT-PCR detection of disseminated tumour cells in bone marrow has superior prognostic significance in comparison with circulating tumour cells in patients with breast cancer. Br J Cancer. 2006;94:672-680.

27. Bolke E, Orth K, Gerber PA, et al. Gene expression of circulating tumour cells and its correlation with tumour stage in breast cancer patients. Eur J Med Res. 2009;14:359-363.

28. Chen CC, Hou MF, Wang JY, et al. Simultaneous detection of multiple mRNA markers CK19, CEA, c-Met, Her2/neu and hMAM with membrane array, an innovative technique with a great potential for breast cancer diagnosis. Cancer Lett. 2006;240:279-288.

29. Lin YC, Chen SC, Hsueh S, et al. Lack of correlation between expression of human mammaglobin mRNA in peripheral blood and known prognostic factors for breast cancer patients. Cancer Sci. 2003;94: 99-102.

30. Liu ZZ, Guo F, Xie XD. Individual detection significances of small breast epithelial mucin (SBEM) and human mammaglobin (hMAM) expressions in peripheral blood of breast cancer patients. ChineseGerman J Clin Oncol. 2012;11:716-720.

31. Grunewald K, Haun M, Urbanek M, et al. Mammaglobin gene expression: a superior marker of breast cancer cells in peripheral blood in comparison to epidermal-growth-factor receptor and cytokeratin-19. Lab Invest. 2000;80:1071-1077.

OncoTargets and Therapy

\section{Publish your work in this journal}

OncoTargets and Therapy is an international, peer-reviewed, open access journal focusing on the pathological basis of all cancers, potential targets for therapy and treatment protocols employed to improve the management of cancer patients. The journal also focuses on the impact of management programs and new therapeutic agents and protocols on
32. Zehentner BK, Persing DH, Deme A, et al. Mammaglobin as a novel breast cancer biomarker: multigene reverse transcription-PCR assay and sandwich ELISA. Clin Chem. 2004;50:2069-2076.

33. Mikhitarian K, Martin RH, Ruppel MB, et al. Detection of mammaglobin mRNA in peripheral blood is associated with high grade breast cancer: interim results of a prospective cohort study. BMC Cancer. 2008;8:55

34. Li C, Zhang T. Human mammaglobin: a specific marker for breast cancer prognosis. J Buon. 2016;21:35-41.

35. Cerveira N, Torres L, Rocha P, et al. Highly sensitive detection of the MGB1 transcript (mammaglobin) in the peripheral blood of breast cancer patients. Int J Cancer. 2004;108:592-595.

36. El-Sharkawy SL, El-Aal WESA, El-Shaer MAEM, Abbas NF, Youssef MF. Mammaglobin: a novel tumor marker for breast cancer. Turkish J Cancer. 2007;37:89-97.

37. Marques AR, Teixeira E, Diamond J, et al. Detection of human mammaglobin mRNA in serial peripheral blood samples from patients with non-metastatic breast cancer is not predictive of disease recurrence. Breast Cancer Res Treat. 2009;114:223-232.

38. Braun S, Cevatli BS, Assemi C, et al. Comparative analysis of micrometastasis to the bone marrow and lymph nodes of node-negative breast cancer patients receiving no adjuvant therapy. J Clin Oncol. 2001; 19:1468-1475.

39. Hassan EM, Willmore WG, McKay BC, DeRosa MC. In vitro selections of mammaglobin A and mammaglobin B aptamers for the recognition of circulating breast tumor cells. Sci Rep. 2017;7(1):14487.

40. Tafreshi NK, Enkemann SA, Bui MM, et al. A mammaglobin-A targeting agent for noninvasive detection of breast cancer metastasis in lymph nodes. Cancer Res. 2011;71(3):1050-1059.

41. Picot N, Guerrette R, Beauregard AP, et al. Mammaglobin 1 promotes breast cancer malignancy and confers sensitivity to anticancer drugs. Mol Carcinog. 2016;55(7):1150-1162.

42. Chaurasiya S, Hew P, Crosley P, et al. Breast cancer gene therapy using an adenovirus encoding human IL-2 under control of mammaglobin promoter/enhancer sequences. Cancer Gene Ther. 2016;23(6):178-187.

43. Goedegebuure PS, Watson MA, Viehl CT, Fleming TP. Mammaglobin based strategies for treatment of breast cancer. Curr Cancer Drug Targets. 2004;4(6):531-542.

44. Zuo L, Li L, Wang Q, Fleming TP, You S. Mammaglobin as a potential molecular target for breast cancer drug delivery. Cancer Cell Int. 2009;9:8.

45. Tiriveedhi V, Fleming TP, Goedegebuure PS, et al. Mammaglobin-A cDNA vaccination of breast cancer patients induces antigen-specific cytotoxic CD4+ICOShiT cells. Breast Cancer Res Treat. 2013;138(1) $109-118$.

46. Tiriveedhi V, Tucker N, Herndon J, et al. Safety and preliminary evidence of biologic efficacy of a mammaglobin-a DNA vaccine in patients with stable metastatic breast cancer. Clin Cancer Res. 2014;20(23): 5964-5975.

47. Kim SW, Goedegebuure P, Gillanders WE. Mammaglobin-A is a target for breast cancer vaccination. Oncoimmunology. 2016;5(2): e1069940.

48. Moher D, Liberati A, Tetzlaff J, Altman DG, The PRISMA Group (2009). Preferred Reporting Items for Systematic Reviews and MetaAnalyses: The PRISMA Statement. PLoS Med 6(7):e1000097.

\section{Dovepress}

patient perspectives such as quality of life, adherence and satisfaction. The manuscript management system is completely online and includes a very quick and fair peer-review system, which is all easy to use. Visit http://www.dovepress.com/testimonials.php to read real quotes from published authors. 